\title{
A 57-year-old man who developed arthritis during R-CHOP chemotherapy for non-Hodgkin lymphoma
}

\author{
F. H. Swaneveld • R. M. van Vugt • J. P. de Boer • \\ B. A. C. Dijkmans - W. F. Lems
}

Received: 2 May 2007 / Revised: 25 June 2007 / Accepted: 2 July 2007 / Published online: 28 July 2007

(C) Clinical Rheumatology 2007

\begin{abstract}
Rituximab is a chimeric human-mouse antiCD20 monoclonal antibody, which is used in the treatment of both B-cell lymphomas and rheumatic diseases. We describe a case of a previously healthy 57-year-old man developing arthritis while being treated with rituximabCHOP chemotherapy (R-CHOP) for a non-Hodgkin lymphoma. The remittant arthritis developed at successively shorter time-intervals after R-CHOP administration and only improved after rituximab was removed from the chemotherapy schedule, suggesting a rituximab-related phenomenon, as extensive diagnostic testing ruled out any other diagnosis.
\end{abstract}

Keywords Adverse effects · Arthritis · Lymphoma ·

Rituximab

\section{Introduction}

Rituximab is increasingly used in the treatment of both Bcell lymphomas as well as rheumatic diseases. Several side-

F. H. Swaneveld · R. M. van Vugt • B. A. C. Dijkmans •

W. F. Lems

Department of Rheumatology, Slotervaart Hospital,

Amsterdam, The Netherlands

\section{J. P. de Boer}

Department of Medical Oncology,

Netherlands Cancer Institute/Antoni van Leeuwenhoek Hospital,

Amsterdam, The Netherlands

\section{F. H. Swaneveld ( $\square)$}

Department of Rheumatology, Slotervaartziekenhuis, Louwesweg 6,

1066 EC Amsterdam, The Netherlands

e-mail: f.h.swaneveld@versatel.nl effects of rituximab are known, but until now only two reports described the development of arthritis after rituximab therapy $[1,2]$. Here we describe yet another case.

\section{Case}

A 57-year-old man was referred to the out-patient clinic of rheumatology because of arthritis of the right knee and left wrist.

The patient had been in perfect health until he noticed a swelling of $2.5 \mathrm{~cm}$ under his right jaw. He was referred to a specialized oncology center where he was diagnosed with an aggressive follicular B-cell non-Hodgkin lymphoma (NHL) stage IVA with involvement of submandibular and mesenteric lymph nodes and bone marrow. Treatment was instituted with R-CHOP chemotherapy, which consists of cyclophosphamide, doxorubicin, vincristine, prednisone, and rituximab, given at a 3-week interval. During this treatment, granisetron (Kytril ${ }^{\circledR}$ ) was given and occasionally magnesiumoxide and paracetamol. Because this patient suffered from a low tumor burden, no tumor lysis profylaxis, i.e., allopurinol was prescribed.

Two and a half weeks after the first course of R-CHOP, the patient developed arthritis of his right knee and left wrist without morning stiffness or involvement of the small hand joints. He had no history of arthralgia, joint swelling, preceding trauma, fever, or infection nor did he have a history of inflammation of the eyes, Raynaud phenomenon, photosensitivity, sicca-syndrome, or inflammatory back pain. The further medical history disclosed several tick bites the year before, with a skin reaction. He rarely used alcohol (maximum of two glasses a day). Family history revealed no rheumatic diseases. 
Physical examination showed a profusely swollen and painful right knee and left wrist. The other joints were not afflicted.

Laboratory testing before the start of chemotherapy showed no abnormalities besides a erythrocyte sedimentation rate (ESR) of $21 \mathrm{~mm} / \mathrm{h}$. At the time of presentation with arthritis, there was ESR of $41 \mathrm{~mm} / \mathrm{h}$, C-reactive protein $256 \mathrm{mg} / \mathrm{l}$, leucocytes $11.1 \times 10^{9} / \mathrm{l}$, a normal liver and kidney function, and an IgM-rheumatoid factor (RF) $7 \mathrm{IE} / \mathrm{ml}$, anti-cyclic citrulline peptide (a-CCP) $<1 \mathrm{AU} / \mathrm{ml}$, anti-nuclear factor (ANF) negative, and urate of $0.25 \mathrm{mmol} / \mathrm{l}$. A diagnostic puncture of synovial fluid from the right knee was performed, which revealed an inflammatory arthropathy (cell count $5,300 / \mathrm{mm}^{3}, 14 \%$ lymphocytes, $8 \%$ mononuclear cells, and $78 \%$ granulocytes) with no signs of malignant cells or crystals. Microbiological testing (Gram-stain, Ziehl-Neelsen, and culture) of the synovial fluid showed no microorganisms. X-rays of both knee and wrist did not show bone involvement or chondrocalcinosis. The patient was treated with naproxen $(500 \mathrm{mg}$, twice daily), with which both pain and swelling of the joints were reduced but not completely resolved.

Four days after the second course of R-CHOP, the pain and swelling returned in all its severity and spontaneously regressed after 1 week.

Shortly after the third course of R-CHOP, the patient experienced the same painful joint swellings, now also involving the left knee. This was in spite of intra-articular kenacort injections and prednisone maintenance therapy (10 mg daily), which was started between the courses of chemotherapy. Repeated pathological and microbiological testing of synovial fluid showed no new results.

After these three courses of R-CHOP, the effect on the NHL was evaluated and disclosed that the intra-abdominal NHL-mass was somewhat reduced, and the remnant of the submandibular lymph node had disappeared.

Since the causal relationship between the (activity of) arthritis and the courses of R-CHOP and rituximab being the only component of R-CHOP of which the development of arthritis is described after the administration of this drug $[1,2]$, the decision was made to give the fourth and fifth course of chemotherapy without rituximab. This time there was no increase in pain or swelling of the joints.

Well over a year after the first R-CHOP chemotherapy, the patient is fully recovered. Evaluation of his NHL shows a near complete remission, and no further treatment was indicated. The arthritis has fully resolved.

\section{Discussion}

Arthritis developing during the course of R-CHOP chemotherapy is very unusual. The arthritis developed after succes- sively shorter time-intervals after the R-CHOP administration while no arthritis occurred when CHOP was given without rituximab. This suggests a probably rituximab related, immune-mediated phenomenon. We were not able to detect antibodies against rituximab or immune complexes that might strengthen this diagnosis. However, we did have strong arguments against the other differential diagnoses.

The pattern of arthritis in this patient is unusual for a rheumatoid arthritis; moreover, both IgM-RF and a-CCP were negative, while $\mathrm{X}$-rays made of the afflicted joints disclosed no erosions.

Spondylarthropathy very rarely develops after the age of 45 years. Moreover, inflammatory back pain was absent, and positive arguments for an infection causing reactive arthritis were lacking.

Other autoimmune diseases like systemic lupus erythematosus (SLE) or Sjögren's syndrome were also very unlikely looking at the clinical presentation, the absence of characteristic signs and symptoms, and the absence of the specific autoantibodies.

A crystalarthropathy is a more likely candidate, as treatment of NHL can cause tumor lysis syndrome, which can give rise to gout. However, serum values of urine acid were repeatedly very low, and there where no other signs of tumor lysis syndrome. Moreover, repeated samples of synovial fluid failed to show any crystals, kidney function was normal; the patient barely used alcohol and did not use diuretics.

The X-rays of both the right knee and left wrist did not show signs of chondrocalcinosis.

Latent infections provoked by the administration of RCHOP were considered. However, repeated cultures, Gramstain, and Ziehl-Neelsen of synovial fluid did not disclose any bacteria or yeasts. Serologic testing for Borrelia Burgdorferi, Lues, Chlamydia trachomatis, Parvovirus B19 and PCR on Mycobacteria tuberculosis and Chlamydia trachomatis (both performed in the synovial fluid) proved to be negative.

A wide spectrum of autoimmune manifestations, among others arthritis, is described in NHL [3]. However, arthritis is unlikely in this patient as autoimmune manifestation, as it developed during R-CHOP and the NHL responded well to this chemotherapy.

Furthermore intra-articular localization of NHL was considered. However, both primary and secondary intraarticular localization of NHL is very rare [4], and the fact that more than one joint is afflicted in this patient makes it even more unlikely. Moreover, magnetic resonance imaging (MRI) of both knees did not show tumor mass, and repeated punctures of synovial fluid of both knees did not show any malignant cells. On top of this, a computed tomography (CT) scan of the abdomen revealed regression of the mesenteric NHL-tumor mass after chemotherapy, 
which means that in the case of any presumed intraarticular localization of NHL, one would expect relapse of joint involvement in the course of chemotherapy instead of progression.

An arthroscopy was considered but not performed because of the risks of infection in this immunocompromised patient, and the low expectancy of achieving the diagnosis by performing the arthroscopy as the presence of NHL intra-articular was considered to be very unlikely.

Because our patient received high dosages of prednisone, the diagnosis of avascular bone necrosis was considered but ruled out by a MRI of both knees. None of the other medications our patient used are known to give arthritis.

Ruling out the above-mentioned diagnoses and taking into account the relationship in time between the development of arthritis and the gifts of R-CHOP, one or more of the components of R-CHOP seem to be the most likely cause of the arthritis in our patient. Of all of the components of R-CHOP, only rituximab is known to be able to give arthritis $[1,2]$.

Pijpe et al. described the development of arthritis in three patients who received rituximab for primary Sjögren's syndrome. All of these patients had developed human antichimeric antibodies (HACAs), which suggests an immune-mediated mechanism. Only one other patient tested positive for HACAs but was asymptomatic. The significance of HACAs being present is actually not quite sure as other studies show HACAs being present without specific clinical manifestations $[5,6]$ or show HACAs being present but later becoming undetectable [6].

Repeated blood tests of our patient did not show any HACAs. The precise mechanism of action in the development of arthritis in our patient is still unknown.
In conclusion, we describe a patient developing severe oligo-arthritis at successively shorter time-intervals after receiving CHOP-rituximab for a NHL. The arthritis is probably caused by an immune-mediated reaction to rituximab. As it is to be expected that rituximab will be used more frequently in future to treat both NHL as rheumatological diseases, we think it is important for physicians to be aware that rituximab may cause severe (oligo-)arthritis as a side effect.

\section{References}

1. Buda-Okreglak EM, Drabick JJ, Delaney NR (2004) Proinflammatory syndrome mimicking acute rheumatoid arthritis in a patient with Waldenstrom's macroglobulinemia treated with rituximab. Ann Hematol 83:117-119

2. Pijpe J, van Imhoff GW, Spijkervet FKL, Roodenburg JLN, Wolbink GJ, Mansour K et al (2005) Rituximab treatment in patients with primary Sjögren's syndrome: an open-label phase ii study. Arthritis Rheum 52(9):2740-2750

3. Jardin F, Levesque H, Tilly H (2005) Auto-immune manifestations in Non-Hodgkin's lymphoma. Rev Med Interne 26(7):557-571

4. Kruger R, Oden J, de Leon F, Durr W (1993) Malignant nonHodgkin's lymphoma of the joint. Report of two cases, review of the literature and problems of classification. Unfallchirurg 96 (10):556-562

5. Edwards JC, Szczepanski L, Szechinski J, Filipowicz-Sosnowska A, Emery P, Close DR et al (2004) Efficacy of B-cell-targeted therapy with rituximab in patients with rheumatoid arthritis. N Engl J Med 350(25):2572-2581

6. Maeda T, Yamada Y, Tawara M, Yamasaki R, Yakata Y, Tsutsumi C et al (2001) Successful treatment with a chimeric anti-CD20 monoclonal antibody (IDEC-C2B8, rituximab) for a patient with relapsed mantle cell lymphoma who developed a human antichimeric antibody. Int J Hematol 74(1):70-75 